\title{
Stage III Adult Diffuse Mixed Cell Lymphoma
}

National Cancer Institute

\section{Source}

National Cancer Institute. Stage III Adult Diffuse Mixed Cell Lymphoma. NCI Thesaurus. Code C8134.

Stage III: Involvement of lymph node regions on both sides of the diaphragm (III) that may also be accompanied by localized involvement of an extralymphatic organ or site (IIIE), by involvement of the spleen (IIIS), or both (IIIS+E). (from PDQ) 